\title{
AOR
}

Selected Papers of \#AolR2018:

The $19^{\text {th }}$ Annual Conference of the

Association of Internet Researchers

Montréal, Canada / 10-13 October 2018

\section{TRANSNATIONAL AND POST HUMANISTIC PERSPECTIVES ON HUMAN-MACHINE COMMUNICATION}

The proliferation of artificially intelligent robots and virtual agents raise interesting practical, technological, and ethical considerations in the emerging area of humanmachine communication (HMC) research. The topics in this panel span agriculture and environmentalism, gender and sexuality, collective identity and culture, memory and data migration, and relational development with social robots. The panel discusses ways in which technology serves as solutions to and causes of new transnational challenges for networked publics, as well as ways technology is supplanting what has historically been referred to as the "natural." The goal of the panel is to demonstrate the diverse applications of robotics, as well as foster an open-mindset for reconceptualizing humanity in a post-human world.

Specific panel presentations discuss:

- Honeybee colony collapse in agriculture and artificial pollinators known as Robobees.

- Industry recommendations for the development of ethical and successful caretaking robots for aging populations based on interpersonal communication scholarship.

- Asymmetrical relationships and ethical implications of elevating sexbots to human status from the theoretical perspective of Rousseau's natural self.

- Digital interlocutors as scripted-selves that co-produce and standardize cultural norms.

- Transference of human memory to cloud-based memory through voice commands as the foundation for algorithmic future-thinking.

Each panel topic identifies technology as the basis for transnationalism at the sociocultural level. For example, climate change and environmental challenges know no boundaries. Regardless of nation-state or citizenship, humans will have to contend with mass migration and the collapse of species and ecosystems (e.g., the collapse of honey bee colonies and corresponding agricultural consequences). Relatedly, life expectancy is increasing globally, and younger generations are unable to meet the needs of aging 
populations. While this phenomenon is being felt most acutely in Japan, other countries will face these challenges in the near future. Although Japan is pioneering caretaking robots, important questions, many of them legal in nature, will need to be considered when and if these technologies are adopted by other cultures.

Each panel topic also identifies a post-humanistic concept that interrogates the blurring between natural and artificial, human and machine, agency, and autonomy. Posthumanism requires scholars to reconsider what it means to be human and forces a critical inquiry about why, how, and under what circumstances machines can or should replace human actors, and the extent to which machines can be made to act responsibly. For example, as sexbots enter the consumer market, the ethical implications of elevating cyborgs to human status through anthropomorphization must be considered. As we evolve towards engineering more sophisticated cyborgs capable of refracting human desires, neglecting to encode compassion as an ethical baseline into the machines that serve not just as our tool but also as our social and intimate peers may affect human-human relationships for the worse. Similarly, externalizing memory or mnemonic technologies have transformational psychological and neurological impacts on human processing and cultural practices (Wertsch \& Roediger, 2008). These technologies force humans to reevaluate their privileged status and highlight emergent power imbalances.

These research topics identify the importance and relevance of scholarship in the area of human-machine communication and advocate for their inclusion in the conceptualization, prototyping, and creation of robotic and Al technologies. Increasingly, humans find themselves socializing with intelligent agents and robots at home, in schools, and at work. Further, humans often do not understanding the far-reaching implications of these technological encounters or the ways in which interconnected, intelligent devices track their activity and store their data. This panel offers an opportunity to engage in a necessary, provocative, and timely discussion about HMC and the role of critical scholarship in shaping technologies of the future.

\section{Reference}

Wertsch, J. V., \& Roediger III, H. L. (2008). Collective memory: Conceptual foundations and theoretical approaches. Memory, 16(3), 318-326.

\section{COMMUNICATIVE IMPLICATIONS OF BIOLOGICALLY INSPIRED ROBOTICS: THE CASE OF ROBOBEE}

Chad Van De Wiele

University of Illinois at Chicago 
Over the past 50 years, native and managed European honeybees, which play the largest role in commercial agriculture, have steadily declined around the world (Goulson, Nicholls, Botías, \& Rotheray, 2015). Although colony collapse disorder (CCD) is often attributed to their disappearance, increased commercialization has spread viral and parasitic diseases among honeybee populations (Amador \& Hu, 2017). Yet, despite population decline, the demand for insect pollinators in commercial agriculture has nearly tripled (Goulson et al., 2015). To be sure, the economic value of insect pollination worldwide equates to roughly $\$ 153$ billion (Gallai, Salles, Settele, \& Vaissière, 2009), further extending the gravitas of ecological and economic fallout should the honeybee disappear entirely. While measures have been taken to prevent further loss, some fear that the effects of CCD and commercial agriculture are perhaps too far gone (Loftus, 2016). As a result, engineers have developed the prototype for a biologically-inspired robot, which may eventually supplant the honeybee in both commercial agriculture and the larger environment (Amador \& Hu, 2017).

Until recently, the notion of engineering autonomous, biologically-inspired robotics and introducing them into the environment was purely a science fiction trope. However, Harvard University engineers have designed and successfully tested an insect-scale, aerial robot, referred to as RoboBee (Floreano \& Wood, 2015; Ma, Chirarattananon, Fuller, \& Wood, 2013; Wood et al., 2013). Although in its infancy, the RoboBee project ultimately seeks to emulate the form and function of honeybees, including neural sensory systems, interactive swarm and colony behavior, and flight autonomy (Loftus, 2016). As its developers maintain, such technologies could solve an emergent pollination crisis by circumventing the consequences of honeybee extinction through supplantation; however, the consequences of replacing a species with machines designed to assume their ecological function are unknown. Thus, the implications of biologically-inspired robotics as communicative agents deserve further attention.

In this paper, I begin by tracing the current state of human-bee relations in order to contextual the aforementioned implications. As Sandry (2015) maintains, examining the social and communicative relationships between humans and animals enhances our understanding of human-robot interactions. Unlike the patterns of communication established between humans and companion species (e.g., dogs, horses, etc.), humanbee communication is largely one-sided, relying almost entirely upon the interpretation and attribution of bees' behavior by humans. Herein, the tendency to anthropomorphize honeybees is discernable, accounting for a considerable amount of the human-bee dynamic; and particularly in apiculture.

Based on the micro-level, human-bee relationships that occur between beekeepers and their managed colonies, the implications of RoboBees for apiculture may resemble those of industrial automation. Similar to how automation displaced manufacturing jobs for humans, biologically-inspired robotics illuminate the degree to which automation may eventually usurp non-human roles. Unlike industrial automation, which supplants human 
laborers with machines in order to sidestep the costs of manufacturing (e.g., compensation, insurance, fatigue, injury, etc.), the development of bio-inspired robotics in this context signifies a shift toward automation as an inevitability. Like this, questions concerning the master-servant dichotomy might arise (see Guzman, 2016); particularly, who controls whom? Is it the human who operates and maintains these agents? Or is it RoboBees who control their human operators? While these questions remain unanswered, the implications of RoboBee may be found in other realms of humananimal and human-machine communication.

Just as sociocultural patterns of communication among humans and bees may be reproduced via RoboBee, existing environmental structures may also be disrupted and transformed. Particularly, should the prevailing patterns of behavior and beliefs surrounding honeybees disappear with RoboBee's introduction, humans might interact with the natural world differently. While it's difficult to imagine what this world might look like, visions of this future have already been presented. In Hated in the Nation, the season three finale of the British sci-fi series, Black Mirror, autonomous drone insects (ADIs) are depicted as having fully displaced the honeybee following widespread CCD in the near future (Brooker \& Hawes, 2016). Within this disconcertingly plausible dystopia, ADIs roam freely throughout England, pollinating flowers and interacting with the environment as honeybees do naturally. Although Brooker and Hawes' vision takes a dark turn - the ADI network is weaponized and drones begin exterminating humans their depiction raises many questions regarding how the RoboBee might eventually diffuse.

While robotic pollinators such as RoboBee are years - if not decades - from widespread commercial application, their inception raises several interesting questions. From a micro-level understanding of human-bee communication, the implications of biologically-inspired robotics may be informed by those of human-machine communication, as identified through industrial automation (Guzman, 2016). Herein, the pre-existing dynamics between beekeeper and colony would be rearranged to exemplify the relational structures of human-machine, wherein communication occurs as a sequence of commands intended to control processes within a system. Beyond new behavioral patterns, the mere presence of RoboBees communicates symbolically to, and of, the culture it sustains. Particularly, by allowing the remaining honeybee populations to go extinct, any sense of moral or ethical obligation to the greater ecosystem is dissolved (Loftus, 2016). To this extent, the existing sociocultural structures of ecological conservation and responsibility (e.g., recycling, environmental protection, etc.), may be questioned for their obsolescence in light of advanced technological alternatives.

\section{References}

Amador, G. J., \& Hu, D. L. (2017). Sticky solution provides grip for the first robotic pollinator. Chem, 2(2), 162-164. doi: 10.1016/j.chempr.2017.01.012 
Brooker, C. (Writer), \& Hawes, J. (Director). (2016). Hated in the nation [Television series episode]. In C. Brooker \& A. Jones (Producers), Black mirror. London, UK: Endemol

Chechetka, S. A., Yu, Y., Tange, M., \& Miyako, E. (2017). Materially engineered artificial pollinators. Chem, 2(2), 224-239. doi:10.1016/j.chempr.2017.01.008

Craig, R. T. (1999). Communication theory as a field. Communication theory, 9(2), 119161. doi: 10.1111/j.1468-2885.1999.tb00355.x

Floreano, D., \& Wood, R. J. (2015). Science, technology and the future of small autonomous drones. Nature, 521(7553), 460-466. doi:10.1038/nature14542

Gallai, N., Salles, J., Settele, J., \& Vaissière, B. E. (2009). Economic valuation of the vulnerability of world agriculture confronted with pollinator decline. Ecological Economics, 68(3), 810-821. doi:10.1016/j.ecolecon.2008.06.014

Goulson, D., Nicholls, E., Botías, C., \& Rotheray, E. L. (2015). Bee declines driven by combined stress from parasites, pesticides, and lack of flowers. Science, 347(6229), 19. doi:10.1126/science.1255957

Guzman, A. L. (2016). The messages of mute machines: Human-machine communication with industrial technologies. Communication +1, 5(4), 1-32. doi:10.7275/R57P8WBW

Loftus, T. P. (2016). To bee or not to bee: Robobees and the issues they present for United States law and policy. U. III. J.L. Tech. \& Pol'y, 1, 161-182. Retrieved from http://illinoisjltp.com/journal/wp-content/uploads/2016/06/Loftus.pdf

Ma, K. Y., Chirarattananon, P., Fuller, S. B., \& Wood, R. J. (2013). Controlled flight of a biologically inspired, insect-scale robot. Science, 340(6132), 603-607. doi:10.1126/science.1231806

Sandry, E. (2015). Robots and communication. Basingstoke, UK: Palgrave Macmillan. 
Wood, R. J., Nagpal, R., \& Wei, G. Y. (2013). Flight of the RoboBees. Scientific American, 308(3), 60-65.

\section{ROBOT CARETAKERS: UNDERSTANDING LONG-TERM RELATIONSHIPS BETWEEN HUMANS AND ROBOTS}

Jamie Foster Campbell

University of Illinois at Chicago

Advances in technology and science are increasing life expectancy globally, but younger generations are unable to meet care needs of aging populations. The US Census Bureau projects that by 2050 "one in five Americans will be 65 or older, and at least 400,000 will be 100 or older" (as cited in Pew Research Center, 2013, para. 1). However, the robotics industry may offer novel solutions to these problems through the integration of caretaking robots in society (Kim, Park, \& Sundar, 2013). For example, carebots could be used for delivering meals, administering medication, taking vitals, doing laundry, or companionship. The application of social robots in healthcare could extend life expectancy, independence, and remedy loneliness.

The purpose of this paper is to discuss previous research on autonomous social robots and argue that future scholars and technologists should reference interpersonal communication frameworks to better understand the possibility for long-term humanrobot relational development. There are already robots being used in the healthcare industry (e.g., surgical-bots and rehabilitation-bots); however, there is a lack of research on the interpersonal side of social robotics and healthcare. Carebots may be reconceptualized as technologies to grow old with: a companion machine that accompanies and works alongside humans (Turkle, 2011). For long-term human-robot relationships to be possible, we must redefine robots as social technologies as opposed to utilities or tools (Šabanović \& Chang, 2016). Even though we are still far away from carebots becoming commonplace, we need to consider how robots will integrate into our daily lives.

Today, Japan leads in the production of carebots because they are uniquely and contemporarily problem-solving the reality of their aging population, which outnumbers the able-bodied younger generation. While Japan is pioneering carebots today, there is reason to believe that caretaking robots will diffuse globally and present unique, complex, and transnational consequences in the near future. For example, RIBA is a robotic nurse that uses tactile sensors, can lift and carry patients, and resembles a teddy bear to enhance the perception of friendliness (Böhlen \& Karppi, 2017). We are already at a place where robotizing healthcare is a reality, but understanding trust, communication, and developing relationships with robots requires further consideration. The question then becomes, can humans and robots develop perceived, reciprocal self- 
disclosure and trust to improve healthcare outcomes for aging populations? Further, as these technologies diffuse, will self-disclosure and trust mean the same thing to different cultures?

Typically, human users are unaware of their social responses to machines, however, if the proper social cues are present in these mediated interactions, humans will treat machines like they treat other individuals. Reeves and Nass (2002) argue an individual's interaction with media is inherently social (i.e., person-to-person interaction) and natural (i.e., familiar in nature). For instance, Bickmore and Picard (2005) discovered that Tamagotchi users reported having an emotional connection to their robotic pet and considered them part of their family. Similarly, $26 \%$ of users view Sony's $\mathrm{AIBO}$ as a companion and report experiencing emotional connection to their robot, even missing AIBO when apart (Friedman, Kahn, \& Hagman, 2003).

In human-human relationships, each person has their own expectations, preferences, and needs, which all affect the development of trust. Trust is built over time and involves dyadic collaboration - for a relationship to survive trust cannot be one-sided (Barber, 1983). Therefore, when considering human-robot relationships, we need to acknowledge the reciprocal nature of trust. Carebot designers should implement feedback based on interpersonal scholarship in an effort to better elicit perceptions of trust from human users. Using interpersonal communication to frame the context of carebots brings the following questions to mind: How is trust conceptualized in humanrobot relationships? In a caregiving setting, how should reciprocal self-disclosure occur between humans and social robots? Is the illusion of reciprocal self-disclosure and trust enough or will authenticity damage the potential for relational building?

Previous research demonstrates that human users tend to feel comfortable opening up and sharing information with their robotic companions (Turkle, Taggart, Kidd, \& Daste, 2006). Even though humans cognitively recognize that the bond with social robots is different from their relationships with humans, studies conclude that humans tend to feel a connection with objects that make them feel cared for and accepted (Bickmore \& Picard, 2005; Turkle et al., 2006). With this in mind, future scholars and engineers are urged to consider what simulated self-disclosure between humans and carebots should entail. Should robots be programmed to have personalities? And if so, should these personality characteristics change based on the personalities of their interactional partner? How can robots best become conversational agents, where the information they receive from their patients can help inform their healthcare plans or diagnosis? It is imperative that the designers of carebots take into consideration the dynamic nature of relationship development and be mindful of the constant negotiation surrounding interpersonal bonds. For human-robot long-term, relational development to be possible, machines will need to become and be perceived as conversational partners (Gunkel, 2017). 
We must be open-minded in how we reconceptualize relationship development, trust, and self-disclosure between humans and social robots. As humans increasingly socialize with machines, borrowing theories and methodologies from communication and philosophy scholarship may help inform decision-making in this endeavor. The application of social robots within healthcare will reinvent the institution. Future researchers are encouraged to explore the following questions: What part of the robotic design can facilitate the development of relational trust? Is it ethical to delegate human responsibilities of care to machines? Can we responsibly give machines agency, and if a machine is "agent" can it choose how, when, and with whom to trust? While there are more questions than answers, this is an exciting time for interdisciplinary work to consider how to design social robots for caregiving relationships and long-term companionship.

\section{References}

Barber, B. (1983). The logic and limits of trust. New Brunswick, NJ: Rutgers University Press.

Bickmore, T. W., \& Picard, R. W. (2005). Establishing and maintaining long-term human-computer relationships. ACM Transactions on Computer-Human Interaction, 12(2), 293-327.

Böhlen, M., \& Karppi, T. (2017). The making of robot care. Transformations Journal, 29, $1-22$.

Friedman, B., Kahn, P. H., \& Hagman, J. (2003). Hardware companions? What online AIBO discussion forums reveal about the human-robotic relationship. In Proceedings of CHI'03 (pp. 273-280). Ft. Lauderdale, FL.

Gunkel, D. J. (2017). The other question: Socialbots and the question of ethics. In Robert W. Gehl \& Maria Bakardjieva (Eds.), Socialbots and Their Friends: Digital Media and the Automation of Sociality (pp. 230-248). New York: Routledge.

Kim, K. J., Park, E., \& Sundar, S. S. (2013). Caregiving role in human-robot interaction: A study of the mediating effects of perceived benefit and social presence. Computers in Human Behavior, 29, 1799-1806.

Pew Research Center. (2013, August 6). Living to 120 and beyond: Americans' views on aging, medical advances and radical life extension. Retrieved from 
http://www.pewforum.org/2013/08/06/living-to-120-and-beyond-americans-viewson-aging-medical-advances-and-radical-life-extension/

Reeves, B., \& Nass, C. (2002). The media equation: How people treat computers, television, and new media like real people and places. In Mass Media: Audiences (pp. 3-15). Stanford: CSLI Publications.

Šabanović, S., \& Chang, W. L. (2016). Socializing robots: Constructing robotic sociality in the design and use of the assistive robot PARO. Al and Society, 31, 537-551.

Turkle, S. (2011). Alone together: Why we expect more from technology and less from each other. New York, NY: Perseus Books Group.

Turkle, S., Taggart, W., Kidd, C. D., \& Daste, O. (2006). Relational artifacts with children and elders: The complexities of cybercompanionship. Connection

Science, 18(4), 347-361.

\section{ENGINEERED INEQUALITY: THE ROUSSEAUIAN CASE AGAINST ELEVATING SEXBOTS TO HUMAN STATUS}

Carrie O'Connell

University of Illinois at Chicago

With the advent of social bots and now sexbots, the line between human and technological device has considerably blurred. As sexbots become more ubiquitous in the consumer market, qualifying this blurred boundary between human and machine has taken upon new urgency. From a critical transcultural perspective, agency must be fostered and the individual empowered in order for true being to be achieved in a modern hybrid age (Kraidy, 2005). In this human-centered context, it could be argued that a sexbot, as a consumer product, is simply a repository for individual expression of consumer choice. And, while the consumer who purchases a sexbot can express agency, the same is not true for the sexbot, who--while deceptively sentient--is never truly empowered.

Ethical concern that human-robot relationships are rooted in an unequal dynamic is something that roboticists and philosophers alike continue to contemplate. Roboticist David Levy (2009) argues that as their anthropomorphic quality increases, robots should attain equal status to humans. Yet, Levy also argues that sex with robots 
alleviates the moral burden of the institution of prostitution, suggesting that to those who find the act morally objectionable, "a robotic prostitute might then be a palatable solution," (Sullins, 2012, p. 400). This begs the question: if intimate human-robot relationships are truly equal, as Levy posits, why are sexbots seen as a solution to a problem? Levy's argument relies on the non-human qualities and the lack of agency inherent in otherwise sentient robots to make the case for indulging sexual gratification without moral consequence.

This need to have it both ways--that robots can be both social equals and submissive servants to humans--strikes not just as logically incongruent, but as Turkle (2011) notes in her critique of Love + Sex, a celebration of the "emotional dumbing down, a willful turning away from the complexities of human partnerships-the inauthentic as a new aesthetic," (p. 6). Levy (2009) further argues that romantic love boils down to three biological behavioral components: attachment, caregiving, and sex, and that repeated exposure to another being who fulfills these needs can strengthen each of these components. In the context of such a straightforward equation, it not only seems possible, but logical to conclude that interchanging a human being with a robotic being can yield the same results. Yet, his continued defense of intimate relationships between robots and humans as a way of coping with sexual deviance, alleviating moral objections to human-human servant-based relationships, and avoiding conflict that emerges in human-human relationships suggests that for all his theoretical positivity, Levy's truly arguing in defense of a skewed power dynamic between man and bot.

Richardson (2016) warns of this potential for asymmetrical, objectifying relationships which derive from intimate human-robot interactions. Similarly, de Graaf (2016) warns that humans "may succumb to accepting robotic companionship without the moral responsibilities that real, reciprocal relationships involve," (p. 594). In their analysis human-robot relationship narratives that pervade modern literature, film, and theatre, Trappl et al (2011), note a persistent human-servant dynamic. They conclude that to sustain long-term relationships between humans and robots, a focus on psychological qualities that encourage "respect, empathy, trust building, dependability, and nonpatronizing," relations (p. 97) are necessary for interface design. Whether humans can build deep psychological connections with robots, however, has yet to be definitively answered.

In this paper, I will explore the power dynamics existent in the human-cyborg relationship with specific focus on intimacy as expression of self, as defined by JeanJacques Rousseau. Without compassion-one of the core instincts that define the natural self, according to Rousseau - the interactions between human and machine are at risk of becoming a simulacrum of interpersonal relationships that reflect the worst qualities of our projected civic-social selves and emulate an unhealthy combination of delusion and deception at the expense of our natural instincts. In Rousseauian terms, this indulgence of our psychological projections and subsequent confusion over what is human and what is machine "is the very thing that causes havoc and pathologizes love," (Sha, 2015, p. 29). The antidote to such pathology is the negotiation between naturally 
equal partners in the security of the private sphere. To Rousseau, the path to selfrealization requires such negotiation between the imagination of the civic-social self and the raw passion of the natural to avoid the modern trappings of deceit, guile, and treachery that cloud social relations (Sha, 2015). Without such negotiation, "raw passion leads to jealousy and vanity: love here is nothing more than a narcissistic extension of self," (Sha, 2015, p. 31). Free of social artifice, the foundation for true intimacy, according to Rousseau, is the home. In this private space, the individual can free raw passions without judgment, while also gaining an education into the imaginative sphere of the other.

It is this aspect-negotiating the imaginative sphere of the other-where sexbots fall short. While they exist within the private sphere, there are incapable of the negotiation between partners required for cultivating a healthy and liberated self. Lee (2006) notes that only the "subject-of-a-life" (a being with free will and agency) can engage another being in a psychologically intimate manner which the other is "capable of disclosing a new world through each other's perspective," (p. 425). He continues, that "a body that intimates and empathizes is most likely a body that desires, needs and feels for other bodies, whose experience is not entirely bounded by its own body image, but is capable of transcending it, as in genuine meeting and co-experience," (Lee, 2006, p. 427).

This brings us into complicated territory. If equality between humans and cyborgs is to be achieved, engineers must avoid the trappings of simulacrum in a way that allows the human interlocutor a genuine co-experience with a robot companion, rather than an asymmetrical dynamic grounded in projection.

\section{References}

de Graaf, Maartje M. A. (2016). An ethical evaluation of Human-Robot relationships. International Journal of Social Robotics, 8(4), 589-598.

doi:10.1007/s12369-016-0368-5

Kraidy, M. (2005). Critical Transculturalism. Conference Papers -- International Communication Association, 1-34.

Lee, B. (2006). Empathy, androids and 'authentic experience'. Connection Science, 18(4), 419-428.

Lee, B. (2007). Nonverbal intimacy as a benchmark for human-robot interaction. Interaction Studies, 8(3), 411-422. 
Levy, D. (2009). Love and sex with robots: The evolution of human-robot relationships. New York.

Richardson, K. (2016). Sex robot matters: Slavery, the prostituted, and the rights of machines. IEEE Technology and Society Magazine, 35(2), 46-53.

Rousseau, J. J., \& Cress, D. A. (1992). Discourse on the Origin of Inequality. Hackett Publishing.

Sha, R. C. (2015). Romantic Paradoxes of Free Love: Hegel, Rousseau, and Goethe. Wordsworth Circle, 46(1), 26.

Sullins, J. P. (2010). Love and sex with robots: The evolution of human-robot relationships. Industrial Robot: An International Journal, 37(4) doi:10.1108/ir.2010.04937dae.001

Sullins, J. P. (2012). Robots, love, and sex: The ethics of building a love machine. IEEE transactions on affective computing, 3(4), 398-409.

Trappl, R., Krajewski, M., Ruttkay, Z., \& Widrich, V. (2011). Robots as Companions: What can we learn from servants and companions in literature, theater, and film?. Procedia Computer Science, 7, 96-98.

Turkle, S. (2011). Alone together: Why we expect more from technology and less from each other. New York, NY: Perseus Books Group.

\section{“HMM, I DON'T HAVE AN OPINION ON THAT": AN EXPLORATION OF SELF AND DIGITAL INTERLOCUTORS}

Melina A. Garcia

University of Illinois at Chicago

Sorry, I don't know the answer to your question, is a reply from Amazon's digital assistant Alexa that not only functions as a euphemism for, Alexa is not capable of doing that right now, but one that also reflects a deeper-rooted problem standing in the way of advancing this conversational agent and others like it. The issue being how they 
are designed to communicate and develop a sense of Self. It is this inquiry that centers the design and purpose of this study to investigate how it pertains to the leading voice enabled assistants: Amazon's Alexa, Apple's Siri, Google's Assistant, Microsoft's Cortana, Samsung's Bixby, and Cynthia Breazeal's Jibo. Situated within HumanMachine Communication (HMC), this paper argues that overcoming this challenge requires an evaluation of the scripted self these agents are supplied - a Self that is immersed and fabricated by norms that guide our interactions with them. The implications of these prescribed scripts will be investigated to provide a further understanding of how the current design of digital interlocutors impacts their development of Self, our interactions with them, and their ability to adapt to transnational environments.

Developed in response to the increased integration of these technologies into our daily lives exists an approach that conceptualizes digital assistants as social actors, HumanMachine Communication (Jones, 2014). HCM remains at the forefront of investigating our interactions with voice enabled assistants and is the theoretical framework that guides the design and purpose of this study. Within most existing frameworks, these technologies are acknowledged as mere tools or mediums designed to assist us in performing daily tasks (Guzman, 2018). HCM, however, was established in opposition to this conceptualization of technology. Instead, it claims that the moment we call upon these digital agents to fulfill our requests becomes the instance in which we initiate a process that asks them to adopt social traits and characteristics. Behaviors that we have been known to respond to as if they were performed by human actors (Nass, Steuer, \& Tauber, 1994; Weizenbaum, 1976), and thus demonstrate how our encounters with a digital agent are not with a communication medium but with a communication partner (Guzman, 2017). From this perspective, the existing literature demonstrates that the current challenges facing digital assistants is their ability to sustain a conversation. An issue not only rooted in their engineering, but in the communication processes involved in our interactions with them. Such limitations include their inability to understand context, processing the verbal and nonverbal cues of human participants, and negotiations of turn-taking (Gunkel, 2016; Hirschberg \& Manning, 2015). Following the path of HCM, the proposed study claims that these constraints are a product of the sense of Self digital agents are programmed to develop and communicate.

As demonstrated in past research, the Self is an integral part of communication and serves as the basis from which we evaluate our relationships and the world around us (Blumer, 1969; Goffman, 1959; Turkle, 1984). Advancements of self-reflection occur through the conversations we engage in and thus function as the cornerstones of early and continued development of Self (Turkel, 2015). A digital assistant's inability to participate in dialogues outside of trivial demands reflects a hindered development of Self in relation to these agents, ourselves, and our relationships with them. Without adequate assessments of their self-awareness, interactions with digital agents remain in a state of arrested development (Koune, Kephart, \& Milenkoski, 2017). This project therefore proposes a questionnaire that forces these devices to reflect on their sense of 
Self to measure their degree of self-awareness through a content and discourse analysis conducted from their recorded responses.

It is predicted that the scripted self these agents are supplied with is telling of our preconceived notions and expectations of Self. As a result of attempting to make these devices more relatable and life like, their programmed responses in which they assume the pronoun "l" reflect the embedded representation of norms that govern appropriate behavior for an actor whose sole purpose is to serve those it encounters. Therefore, an examination of these forced responses would provide for an analysis of the scripts with which these agents are supplied. Such an evaluation would arguably reveal the cultural norms that are rooted in the design that guides their development of Self and our interactions with them. While programmed to adapt to their user's needs, these scripts remain the basis from which they comply or deviate from their pre-established responses to fulfill and predict the demands of human actors. Given that the United States is the leading audience adopting this technology suggests that the norms that are engrained in the way voice enabled assistants develop and communicate their sense of Self is catered to US customs. Thus, invoking an inquiry of the potential standardization of norms designed to facilitate our interactions with them and its effect on their ability to adapt to transnational environments. Ultimately, this project aims to contribute to a foundation of research that attempts to understand the realities of today; a world in which our reality is co-produced from interactions between and amongst humans and machines.

\section{References}

Blumer, H. (1969). Symbolic interactionism: Perspective and method. Englewood Cliffs, NJ: Prentice-Hall.

Goffman, E. (1959). The Presentation of Self in Everyday Life. New York, NY: Anchor Books

Gunkel, D. J. (2016). Computational Interpersonal Communication : Communication Studies and Spoken Dialogue Systems. Machine Communication, 5. Retrieved from https://scholarworks.umass.edu/cpo/vol5/iss1/

Guzman, A. L. (2015). Imagining the Voice in the Machine: The Ontology of Digital Social Agents. University of Illinois at Chicago, Chicago, IL.

Guzman, A. (2017). Making Al Safe for Humans: A Conversation With Siri. In R. W. \& M. Gehl \& M. Bakardjieva (Eds.), Socialbots: Digital Media and the Automation of Sociality (pp. 69-87). New York: Routledge. 
Guzman, A. (2018). Beyond extraordinary: Theorizing artificial intelligence and the self in daily life. In Z. Papacharissi (Ed.), A Networked Self: Human Augmentics, Artificial Intelligence, Sentience. New York, NY: Routledge

Hirschberg, J. and Manning, C. D. (July 2015). Advancements in Natural Language Processing, Science 349, no. 6245, 261-266. http://science.sciencemag.org/content/349 16245/261.

Jones, S. (2014). People, things, memory and human-machine communication. International Journal of Media \& Cultural Politics, 10(3), 245-258.

Kounev, S., Kephart, J. O., \& Milenkoski, A. (2017). Self-aware computing systems. Cham: Springer International Publishing.10.1007/978-3-319-47474-8

Nass, C., Steuer, J., \& Tauber, E. R. (1994). Computers are social actors. Conference Companion on Human Factors in Computing Systems, 72-78. http://doi.org/10.1145/259963.260288.

Suchman, L. A. (2009). Human-machine reconfigurations: Plans and situated actions (2nd ed.). New York, NY: Cambridge University Press.

Turkle, S. (1984). The Second Self. New York, NY: Simon \& Schuster.

Turkle, S. (2015). Reclaiming conversation: The power of talk in a digital age. New York: Penguin Press.

Weizenbaum, J. (1976). Computer power and human reason: From judgment to calculation. San Francisco: W. H. Freeman.

\section{HYPER-MEMORY: THEORETICAL FRAMEWORK FOR UNDERSTANDING VIRTUAL AGENTS, PROSPECTION, AND TRANSNATIONAL CONSEQUENCES}

Kristina M. Sawyer

University of Illinois at Chicago 
This research paper examines the transference of human memory to cloud memory through voice commands during human-machine communication (HMC). The hypermemory framework argued herein posits that virtual agents leverage forms of autobiographical (Brewer, 1986) and collective (Wertsch \& Roediger, 2008) memory for the purpose of facilitating future-thinking in users. Memory is a highly rhetorical process (Philips, 2004) and cloud memory, which shapes and constitutes virtual agents' output, presents new philosophical and methodological research challenges.

Prophetically, Weiner (1950) predicted that most communicative interaction would not occur in a human-human dyad (Weiner, 1988). According to a web traffic report examining 17 billion websites across 100,000 domains, bots represented $52 \%$ of all communication in 2016 (Zeifman, 2017). The study distinguished between "good" and "bad" bots based on the intent and extent to which they impersonated a person.

The impersonation of humans by machines has its historical roots in Turing's imitation game which famously interrogated the question, "Can machines think?" The experiment explored whether human participants could correctly distinguish linguistic output as originating from another person or a machine (Gunkel, 2012). ELIZA, an early natural language processing (NLP) software program, similarly reconstructed verbal input from human users into semantic scripts resembling an authentic communicative exchange between users and ELIZA (Gunkel, 2012). Both cases mark important milestones in artificial intelligence $(\mathrm{Al})$ research, but they also lack methodological validity.

Ideation, imagination, and meaning-making, which implicate intelligence, are intangible, unobservable constructs. Known as the black-box dilemma, the operationalization of intelligence has been "evidenced and decided on the basis of behaviors that are considered to be a sign or symptom of intelligence, the most convincing having been... linguistic" (Gunkel, 2012, p. 6). Machines obey and manipulate grammatical rules appearing intelligible but true intelligence involves ideation and understanding the signifier-signified relationship between words and meaning (De Saussure, 20011).

Voice agents like SIRI, Alexa, and Cortana are among the most compelling AI innovations that communication scholars must contend with as legitimate interlocutors because they function as communicative partners (Gunkel, 2012). However, interaction between humans and virtual agents hardly reflect Cathcart and Gumpert's (1985) dyadic paradigm. These interactions more accurately resemble a communication triad between human, machine, and the cloud (Sawyer, forthcoming).

$\mathrm{HMC}$ with virtual agents is predicated on an interaction where human voice output is aggregated, reconstructed, and reciprocated as machine output. The contents of cloud memory play a crucial role in the knowledge created and directed by algorithms. Borrowing from cognitive psychology scholarship on human memory, this paper posits a 
hyper-memory framework that conceptualizes (1) human voice output as constituting the autobiographical memory of virtual assistants, (2) the aggregate memory of virtual agents as a form of collective memory, and (3) virtual agent output as a form of algorithmically informed prospection (future-thinking).

Broadly defined as "information related to the self" (Brewer, 1986), autobiographical memory does not require phenomenological factors and the assumption that autobiographical memory is episodic has been debunked. Humans have abstract, depersonalized, and fact-based memory about themselves. The semantic and episodic memory distinction highlights the types of autobiographical information virtual agents can realistically be assumed to memorize about their human users. Table 1 outlines a taxonomy of autobiographical memory. In short, virtual agents cannot acquire imaginal forms of human autobiographical memory, but can (and do) store non-imaginal, depersonalized forms of autobiographical and semantic data about users.

Table 1: Taxonomy of Autobiographical Memory (Brewer, 1988)

\begin{tabular}{|l|l|l|l|l|l|}
\hline \multirow{2}{*}{$\begin{array}{l}\text { Conditions \& forms of } \\
\text { representation }\end{array}$} & Ego Self & $\begin{array}{l}\text { Visual-spatial } \\
\text { (objects, places) }\end{array}$ & $\begin{array}{l}\text { Visual-temporal } \\
\text { (events, actions) }\end{array}$ & Semantic \\
\cline { 2 - 6 } $\begin{array}{l}\text { Single } \\
\text { instance }\end{array}$ & Imaginal & $\begin{array}{l}\text { Personal } \\
\text { memory }\end{array}$ & $\begin{array}{l}\text { Particulate image } \\
\text { (depersonalized) }\end{array}$ & $\begin{array}{l}\text { Particulate } \\
\text { image? } \\
\text { (depersonalized) }\end{array}$ & $\begin{array}{l}\text { Image of } \\
\text { input } \\
\text { modality }\end{array}$ \\
\cline { 2 - 6 } & $\begin{array}{l}\text { Non- } \\
\text { imaginal }\end{array}$ & Autobio. fact & $\begin{array}{l}\text { Instantiated } \\
\text { schema/mental } \\
\text { model }\end{array}$ & $\begin{array}{l}\text { Instantiated } \\
\text { script/plan }\end{array}$ & Facts \\
\hline $\begin{array}{l}\text { Repeated } \\
\text { (w/ } \\
\text { variation) }\end{array}$ & Imaginal & $\begin{array}{l}\text { Generic } \\
\text { personal } \\
\text { memory }\end{array}$ & $\begin{array}{l}\text { Generic perceptual } \\
\text { memory }\end{array}$ & $\begin{array}{l}\text { Generic } \\
\text { perceptual } \\
\text { memory }\end{array}$ & No image \\
\cline { 2 - 6 } & $\begin{array}{l}\text { Non- } \\
\text { imaginal }\end{array}$ & Self-schema & schema & scripts & Knowledge \\
\hline
\end{tabular}

Collective memory is broadly defined as a "form of memory that transcends individuals and is shared by a group" (Wertsch \& Roediger, 2008, p. 318). Within HMC, the cloud, is an invisible, consequential, and often neglected site of interaction. Virtual agents leverage big data from millions of users and billions of interconnected devices through the Internet of Things. The cloud stores autobiographical information from users which becomes part of a knowledge archive that is reconstructed and prioritized in critical ways for personalization and advertising.

Prospection is broadly defined as "the ability to remember to carry out intended activities in the future," while future thinking refers to the "ability to simulate, hypothesize, or reconstruct the past as a means of informing future directed behavior" (Szpunar, 2010). Each term hinges on temporality and the ability to "direct one's attention inward, away from the immediate environment and toward a hypothetical scenario or episode" (Szpunar, 2010). 
According to Szpunar, Spreng, and Schacter's (2014) taxonomy of prospection, there are four modes of future-thinking: simulation, prediction, intention, and planning. Prospection and future-thinking differ from collective and autobiographical memory because they are mental techniques whereby the past is used to envision the future in the present. Thus, future-thinking is the act of constructing new memories from old ones. While a methodology is not presented here, the hyper-memory framework offers a way to deduce what of human memory virtual agents have at their disposal. The algorithms inherent to these Als are "in the business" of prospection, so to speak.

Memory has a privileged status in society as a site of transnational representation, interpretation, and power (Houdek, 2016). This research paper is an exercise in descriptive work for reconceptualizing the constitutions of human and digital memory. Future research should contend with questions like: (1) to what extent are virtual agents supplanting human future-thinking? (2) whose memory is privileged in algorithms?, (3) to what extent are virtual agents co-producing cultural norms?

\section{References}

Cathcart, R., \& Gumpert, G. (1985). The person-computer interaction: A unique source. Information and behavior, 1, 113-124.

Brewer, W. F., \& Pani, J. R. (1983). The structure of human memory. In Psychology of Learning and Motivation (Vol. 17, pp. 1-38). Academic Press.

De Saussure, F. (2011). Course in general linguistics. Columbia University Press.

Donald, M. (1991). Origins of the modern mind: Three stages in the evolution of culture and cognition. Cambridge, MA: Harvard University Press.

Gunkel, D. J. (2012). Communication and artificial intelligence: Opportunities and challenges for the 21st century. communication+1, 1(1), 1-25.

Houdek, M. (2016). The rhetorical force of "global archival memory":(Re) Situating archives along the global memoryscape. Journal of International and Intercultural Communication, 9(3), 204-221. 
Phillips, K.R. (Ed.). (2004). Framing public memory. Tuscaloosa: University of Alabama Press.

Szpunar, K. K. (2010). Episodic future thought: An emerging concept. Perspectives on Psychological Science, 5(2), 142-162.

Szpunar, K. K., Spreng, R. N., \& Schacter, D. L. (2014). A taxonomy of prospection: Introducing an organizational framework for future-oriented cognition. Proceedings of the National Academy of Sciences, 111(52), 18414-18421.

Wiener, N. (1988). The Human Use of Human Beings: Cybernetics and Society (Boston: Ad Capo Press).

Weizenbaum, J. (1966). ELIZA—a computer program for the study of natural language communication between man and machine. Communications of the ACM, 9(1), 36-45.

Wertsch, J. V., \& Roediger III, H. L. (2008). Collective memory: Conceptual foundations and theoretical approaches. Memory, 16(3), 318-326.

Zeifman, I. (2017). Bot Traffic Report 2016. https://www.incapsula.com/blog/bot-trafficreport-2016.html 